\title{
Comparative study of the effect of PEG and Crodamide on the scratch resistance of PMMA
}

\author{
M. Mansha ${ }^{1}$, C. Gauthier ${ }^{1}$, P. Gerard ${ }^{2}$ \& R. Schirrer ${ }^{1}$ \\ ${ }^{1}$ University of Strasbourg, Institute Charles Sadron, CNRS, France \\ ${ }^{2}$ Arkema, GRL Lacq, France
}

\begin{abstract}
Scratch durability of polymer surfaces and coatings is becoming crucial for the growing use of these materials in innovative applications, replacing former materials with more resistant surfaces. A variety of physico-chemical processes such as annealing and a range of ion plantation techniques have been proved very valuable for the renovation of polymeric surfaces but their applications are restricted to some certain polymers due to a number of disadvantages. In particular, several of them can not be applied to translucent polymers like PMMA due to their darkening outcome. Enhancement of the scratch resistance must be investigated for the most part as a consequence due to decreasing the friction coefficient. The recovery increases if the tip is smooth or if the local friction coefficient is low. Discovery of appropriate plasticizers for different polymers to decrease their surface friction is yet to be made in order to improve their scratch resistance.

The effect of PEG and crodamide on both surface properties and bulk mechanical properties of PMMA was studied over a wide range of temperature (ranging from -40 to $+85^{\circ} \mathrm{C}$ ). Our experimental results show that a decrease in friction coefficient is possible by the introduction of appropriate plasticizer without having a significant effect on its bulk behaviour and this decrease in friction depends upon the nature and the content of plasticizer. Moreover, fatty acid amides have been proved more effective in decreasing friction than PEG. The friction decreases directly with the percentage of crodamide. The values of the rear contact angle and in-situ photographs during scratching confirm that the decrease in friction is associated with the decrease of yielding of the contact between the tip and the polymer surface.
\end{abstract}

Keywords: scratch resistance, friction coefficient, polymer, PMMA, crodamide, PEG, bulk properties. 


\section{Introduction}

Polymers are becoming more and more attractive for a growing number of industries. Their low cost, ease and simplicity of manufacture and processing, low weight as well as the attractive look they furnish to any surface have amplified their use [1-5]. Abrasion and scratching are by and large the cause of the loss of their optical and tribological performances [6-9]. The surfaces of polymeric materials are predominantly sensitive to scratches, which obstruct their use for high performance optical devices. In other state of affairs, these materials are used for moving mechanical parts, where friction and wear properties are important [10]. Scratches on plastic surfaces seriously trim down the surface aesthetics of the polymer products and also lead to stress concentration, making products prone to premature failure. That is why there is a strong call for to get better scratch resistance of polymers for automotive and other applications that insist high surface excellence $[3,11]$.

It is normally acknowledged that surface damage can be divided into two types; mar and scratch. A mar is a spot due to a sliding body that is excessively low to be ostensible by the casual human eyes alone but however does turn into noticeable when present in huge quantities. The classic damage found on paint coats and dashboard surfaces smashed by tiny objects such as rough stones, sticks, keys, etc are the examples. A scratch is a mark that makes perceptible grooves and/or surface damage; this is the distinctive damage mode for surfaces that endure profound moving loads by swivels, ball bearings, etc. [2, 12]. The asperities on the surface of a hard material, if they come in contact, can easily generate scratches on the polymer surface. The infrequent occurrence of hard debris or third-body, as they are referred to, can also be a cause of scratches on the polymer surfaces [13]. Scratches may be of any size and length, and the perceiving, or valuation, of the scratch is generally linked with its look [11].

Scratch method offers an appropriate and trustworthy way to study the mechanical properties of organic polymers under different contact conditions [6, 10, 11, 13-16]. A number of researchers have used scratch test to evaluate resistance to scratch for different polymers. Owing to the large elastic recovery of polymers and their complex rheological behaviour, the analysis and the elucidation of this test, however, is far more complicated than for metals [14]. Chief variables that can modify the damage response of polymers during scratching are strain (defined by the angle of attack), scratching velocity, normal load (that determines the depth of scratch), temperature or thermal effects, state of lubrication and the stiffness property of the scratching device. In order to slim down various effects, Briscoe et al. [11] have proposed the idea of using scratching maps while presenting scratching data. Although qualitative in nature, scratching maps provide an outstanding way of identifying some broad deformation behaviour of polymers such as ductile, brittle or visco-plastic for a broad array of experimental parameters $[13,15]$.

Jardret et al. have revealed three kinds of deformation during the experiments: plastic or stable deformation, surface elastic deflection in the region of the contact zone, and the elastic recovery of the scratch groove. Measurement of the 
scratch width permits the assessment of the contact depth and therefore of the contact pressure between the indenter and the material's surface [16]. Sue and coworkers have concentrated to apply the scratch test for evaluating the surface scratch resistance of different polymers. They noticed that the scratch process is a function of the basic material properties such as the elastic modulus, the yield strength and the tensile strength as well as material surface characteristics such as the coefficient of friction $[2,3,15]$.

The contact area between a moving tip and a polymer surface has a front side and a rear side and its shape changes with the strain [12, 17, 18]. A contact area which is totally plastically deformed is called for simplicity a 'viscoplastic contact'. If the contact area is not entirely plastically deformed, it is called a 'viscoelastic-plastic contact'. The shape of the contact is simply described by the rear angle or by the ratio of the rear length to the front length. The rear contact angle $\omega$ is due to the elastic recovery of the polymer and depends on the plastic deformation around the contact. Figure 1 gives a schematic illustration of the evolution of the rear contact angle where as Figure 2 represents contact angle as a function of the mean contact strain $\varepsilon . \quad \varepsilon_{\mathrm{e}}$ and $\varepsilon_{\mathrm{p}}$ represent the contact strains at the end of the elastic contact area and the commencement of the plastic contact area, respectively. In the case of a viscoelastic contact, the rear angle $\omega$ is equal to $\pi / 2$ for the elastic deformation. If the material shows strain hardening, the contact strain decreases and $\omega$ increases. As the strain rate and temperature vary, even under constant loading, the contact area may also vary significantly and the strain in the surrounding area of the contact may change from viscoelastic to viscoplastic $[17,18]$. The material underneath a moving tip may demonstrate diverse types of behaviour: elastic, elastic-plastic where elastic and plastic strains are present in the contact area, or fully plastic. As is accustomed in polymers, the material behaviour is time and temperature dependent and may display strain hardening $[18,19]$.

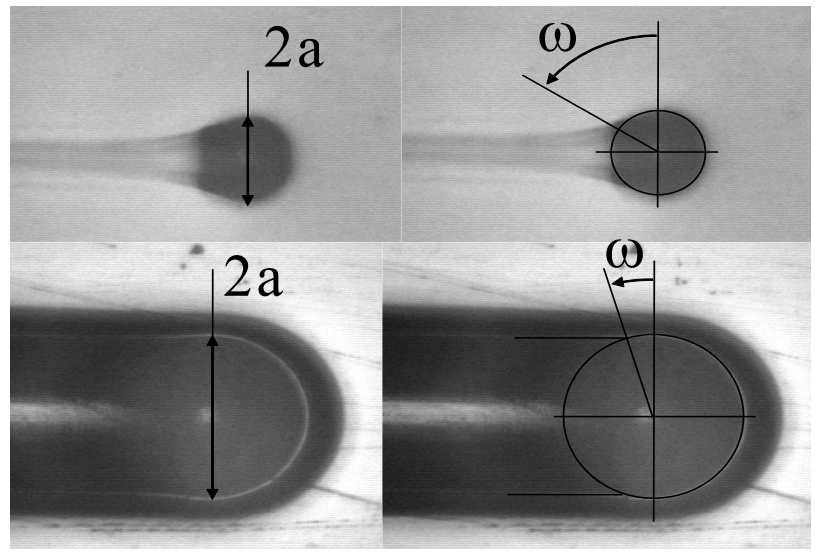

Figure 1: Photograph of the contact area during scratching. This area has a rear part defined by the angle $\omega$. 


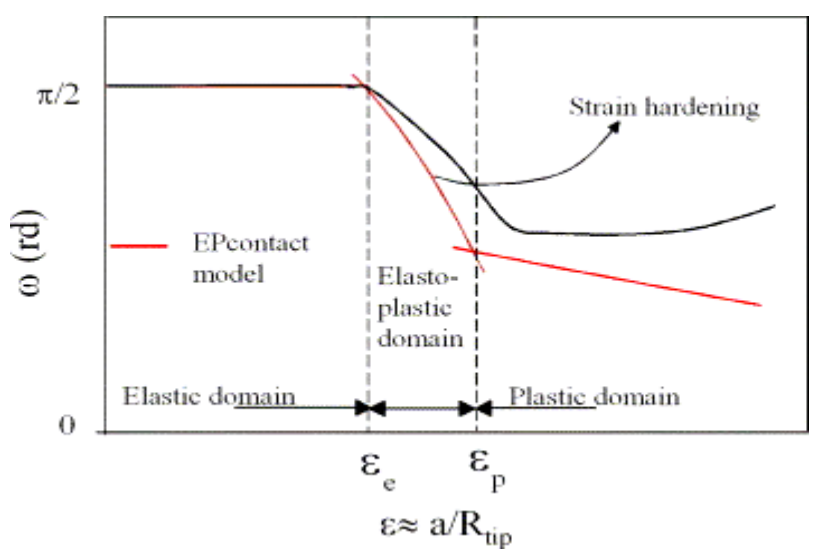

Figure 2: $\quad$ Rear contact angle as a function of the mean contact strain. This angle decreases in the case of a viscoelastic-plastic or viscoplastic contact and increases if the material shows strain hardening [17, $18]$.

Another important property that is universally studied by means of a scratch test is the overall friction coefficient defined as the ratio of the tangential force to the normal force. It is normally believed to take place from two mechanisms: adhesion and ploughing. It has been found that the overall friction coefficient in a scratching test is influenced by many factors such as material type, sliding speed, lubricating conditions, and the indenter geometry. The experimental results by Briscoe et al. indicated that the ratio of adhesive interfacial force to the total scratch force tended to unity when the cone angle of the indenter was increased in single-pass scratch tests. This may also imply that the ploughing effect decreases with the increasing cone angle of the indenter [20]. The polymer true friction coefficient displays temperature and sliding speed dependency, which may be recognized due to the surface thermodynamics. It was shown by Lafeye et al. that the local friction depends on the level of strain in the polymer at the contact interface $[18,19]$.

Gauthier et al. observed the transition from viscoplastic scratching to elastic sliding and found that the temperature, strain and strain rate are the important parameters controlling the nature of scratching on viscoelastic viscoplastic materials like polymers [10]. They proved for the macroscopic scale that the mechanical performance of the contact is tailored by the decrease in the friction coefficient. They also presented an analysis of the elastic recovery of a scratch after contact with a tip. They concluded that the recovery increases if the tip is smooth or if the local friction coefficient is low. So the upgrading of scratch resistance should be investigated principally as an outcome caused by decreasing the friction coefficient [17]. Exploration of the reduction of friction through use of different plasticizers is an area that has an immense potential of research. Plasticizers are normally used to perk up flexibility, trim down cost and improve the processability of polymers. Preferably, plasticizers would be low in price, 
compatible with the host polymer and be processed without difficulty. For extending the life span of plasticized materials, the additive ought to have a low volatility and should not seep from the host. The plasticizer should also give reproducible control over material properties, by adjusting the chemical structure and the quantity of the plasticizer used [21].

\section{Experimental details}

\subsection{Materials and methodology}

\subsubsection{Materials}

The PMMA studied was a standard grade PMMA (V825), having molecular weight about $100 \mathrm{~K}$. It was provided by Arkema. The effect of plasticizers was studied by testing the samples containing $0,1,3$, and $5 \%$ of either of the both; i.e. PEG and Crodamide. PEG is generally recognised due to its low cost and antiblock properties [22]. Owing to compatibility with most of polymers, fatty acid amides are being used world widely to overcome many of the slip, blocking and mould-release problems faced by the polymer industry [23]. Crodamide, like other fatty acid amides, has superior thermal stability which permits its use at relatively higher process temperatures where use of other conventional additives is not recommended [24, 25]. PEG $\left[\left(\mathrm{C}_{2} \mathrm{H}_{4} \mathrm{O}\right) \mathrm{nH} 2 \mathrm{O}\right]$ employed had Mol. Wt. 3350 and was purchased from Sigma-Aldrich where as Crodamide used was Crodamide ER (or refined erucamide) and was purchased from Croda Chemicals.

\subsubsection{Scratching apparatus and the indenter}

The experimental data was obtained with a scratch apparatus fitted with a built-in microscope, which allows in situ examination of the contact area and the groove left on the surface (which is practicable because of the lucidity of the tested polymers). The scratch apparatus, described in detail by Gauthier and Schirrer, is known as the microvisioscratch apparatus. It is based on a commercial servomechanism having a small translucent environmental compartment, which contains the sample and the moving tip (Figure 3). Scratch test may be performed over a large array of speeds $(1 \mu \mathrm{m} / \mathrm{s}$ to $15 \mathrm{~mm} / \mathrm{s})$ and in a temperature range covering both $\alpha$ and $\beta$ transitions of all-purpose polymers $\left(-70^{\circ} \mathrm{C}\right.$ to $+120^{\circ} \mathrm{C}$ ). The normal load applied to the tip can be varied from $0.05 \mathrm{~N}$ to $35 \mathrm{~N}$. Control of the moving tip and the recording of the load, the speed of the sliding tip and the temperature are computer driven. The tip starts at the lowest value of the velocity (or at the lowest value of normal load in the alternate mode) and accelerates stepwise up to the highest value. At each step, it moves over a distance of at least $1 \mathrm{~mm}$ so as to get a groove that can be easily measured and photographed in-sit. The input parameters are the normal load, tip geometry, temperature and the sliding speed, whereas the output parameters are the tangential force, groove geometry and the true contact area.

All samples were scratched by means of a conical scratcher (tip radius $=0.116$ $\mathrm{mm}$ and apex angle $=60^{\circ}$ ) with a varying load from $0.05 \mathrm{~N}$ to about $5 \mathrm{~N}$ 

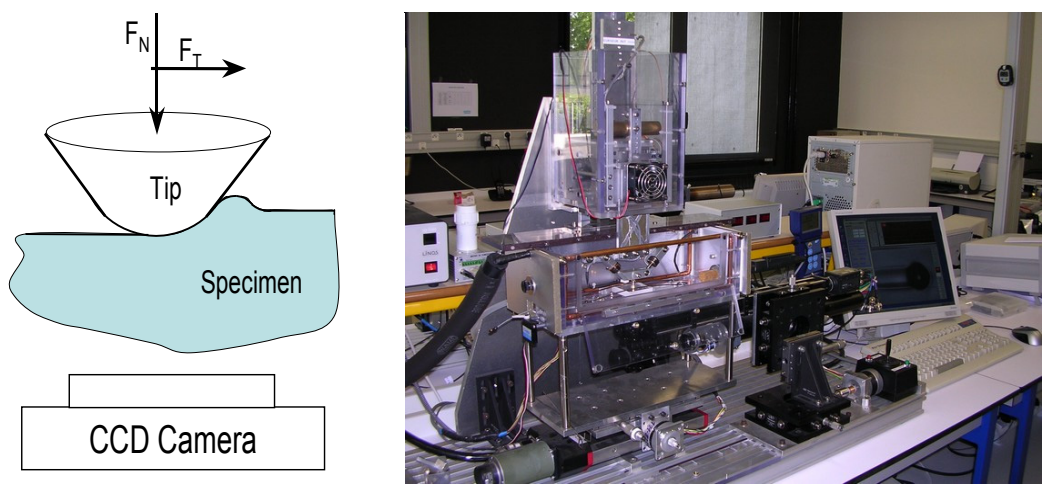

Figure 3: A schematic diagram (on the left) and the photograph (on the right) of the friction measuring apparatus.

depending upon the test temperature (ranging from $-40^{\circ} \mathrm{C}$ to $85^{\circ} \mathrm{C}$ ). The scratcher was made of diamond, and the sliding speed was maintained at about $0.03 \mathrm{~mm} / \mathrm{s}$. A typical procedure was used to perform the friction tests [19]. Sliding and scratching tests are characteristically carried out at mounting normal load at a constant speed for a given temperature. At every loading step, the true contact area is photographed in situ. This image helps one to find out the shape and width of the contact area [26].

\subsubsection{Measurement of bulk properties}

All the specimens with different level of plasticizer content were tested using Instron 4015 Universal Testing Machine with capacity of $10 \mathrm{kN}$ and ram velocity ranging from $1.0 \mu \mathrm{m} / \mathrm{sec}$ to $10.0 \mathrm{~mm} / \mathrm{sec}$. The main objectives of the tests are to obtain the values of tensile strength and strain, shear stress and strain at break, elongation at breakage and the relation of these values versus plasticizer contents for PMMA.

\section{Results and discussion}

\subsection{Surface properties}

\subsubsection{Coefficient of friction}

As discussed in section 1 , the apparent friction coefficient $\left[\mu_{\mathrm{app}}\right]$ is the proportion between the tangential force and the normal load applied to the moving body in contact with the surface of a material. This coefficient includes a so-called "true local friction" $\left[\mu_{\mathrm{loc}}\right]$ - also named as "adhesive friction" $\left[\mu_{\mathrm{ad}}\right]$ in case of perfectly smooth surface and a "geometrical friction" which is the ploughing effect. The latter may be decomposed into two terms; one term due to the viscoelasticity and the other due to the plasticity. Hence the friction may be written as $[12,18,19]$.

$$
\mu_{\mathrm{app}}=\mu_{\mathrm{ad}}+\mu_{\mathrm{visco}}+\mu_{\mathrm{plast}}
$$


When a slip agent (a plasticizer being used for reducing the friction) is mixed in a polymer melt, it is absorbed in polymer region. It remains there as long as polymer remains in melt form. When the polymer solidifies at lower temperature, the additive becomes incompatible with the polymer due to difference in surface energy and start migration towards surface. Fatty acid amides are characterised for forming a thin film over the surface of the finished product giving an excellent slip effect [22]. Our experimental results indicate that the crodamide is more effective than PEG in reducing friction coefficient of PMMA (Figures 4 and 5).

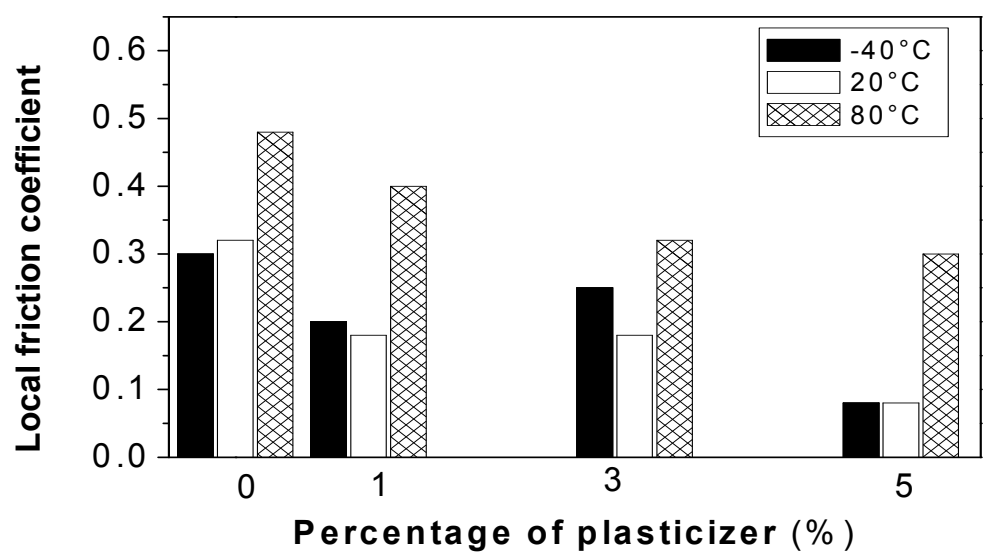

Figure 4: Effect of crodamide on local friction coefficient of PMMA at $40^{\circ} \mathrm{C}, 20^{\circ} \mathrm{C}$ and $80^{\circ} \mathrm{C}$ (sliding speed $=0.03 \mathrm{~mm} / \mathrm{sec}$, sliding tip radius $=116 \mu \mathrm{m}$, normal load $=1 \mathrm{~N})$.

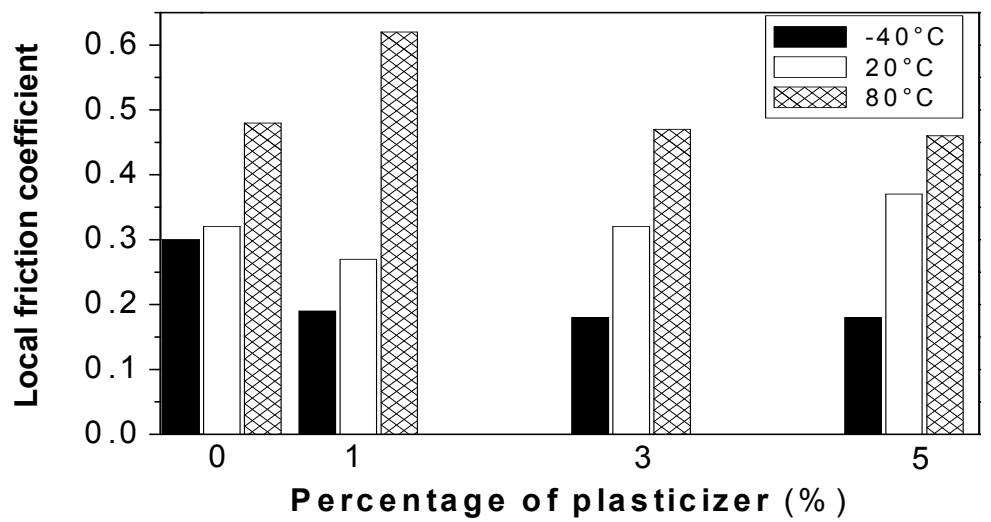

Figure 5: $\quad$ Effect of PEG on local friction coefficient of PMMA at $-40^{\circ} \mathrm{C}$, $20^{\circ} \mathrm{C}$ and $80^{\circ} \mathrm{C}$ (sliding speed $=0.03 \mathrm{~mm} / \mathrm{sec}$, sliding tip radius $=116 \mu \mathrm{m}$, normal load $=1 \mathrm{~N})$. 
The decrease in friction coefficient increases with increasing crodamide content. On the other hand, in case of PEG, the coefficient of friction decreases initially on the introduction of PEG (up to $1 \%$ ) but, on further addition of PEG, no additional reduction in the friction coefficient is observed. Moreover, at higher temperature, no any significant effect of addition of PEG on the reduction of the friction coefficient of PMMA is noticed. Due to higher molecular weight of PEG, it is difficult to be migrated to the surface and to form a film lowering the friction. The values of bulk properties like young modulus of the two cases (as shown in next section) also support this conclusion.

\subsubsection{Contact angle}

As discussed in section 1, the contact angle (omega) is the indicator of the nature of the contact between the sliding tip and the polymer surface. Our experiments indicate that the introduction of crodamide in PMMA modifies the nature of the contact and enhances the visco-elasticity in the contact. If we see, for example, the values of contact angle for the samples containing different percentages of crodamide at room temperature (Figure 6), we come to know that at same value of contact strain (0.05), the value of omega is changed significantly by the introduction of crodamide. It is about 0.4 for pure PMMA (having higher value of local friction coefficient) that increases to 0.9 for crodamide -plasticized PMMA (having lower value of local friction coefficient). So it can be concluded that the decrease in friction coefficient is associated with the increased value of the contact angle and ultimately with the nature of the contact. In situ photographs of the contact demonstrate that the contact nature is plastic for pure

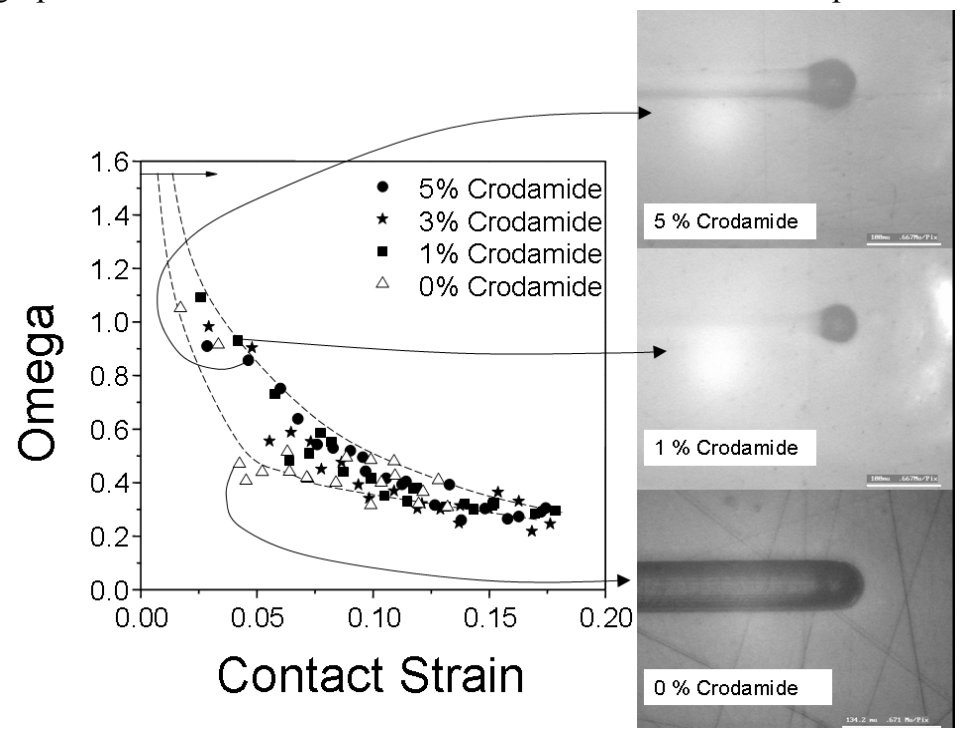

Figure 6: Variation of omega with contact strain and the percentage of Crodamide for PMMA at room temperature (sliding/scratching speed $=0.03 \mathrm{~mm} / \mathrm{sec}$, tip radius $=116 \mu \mathrm{m})$. 


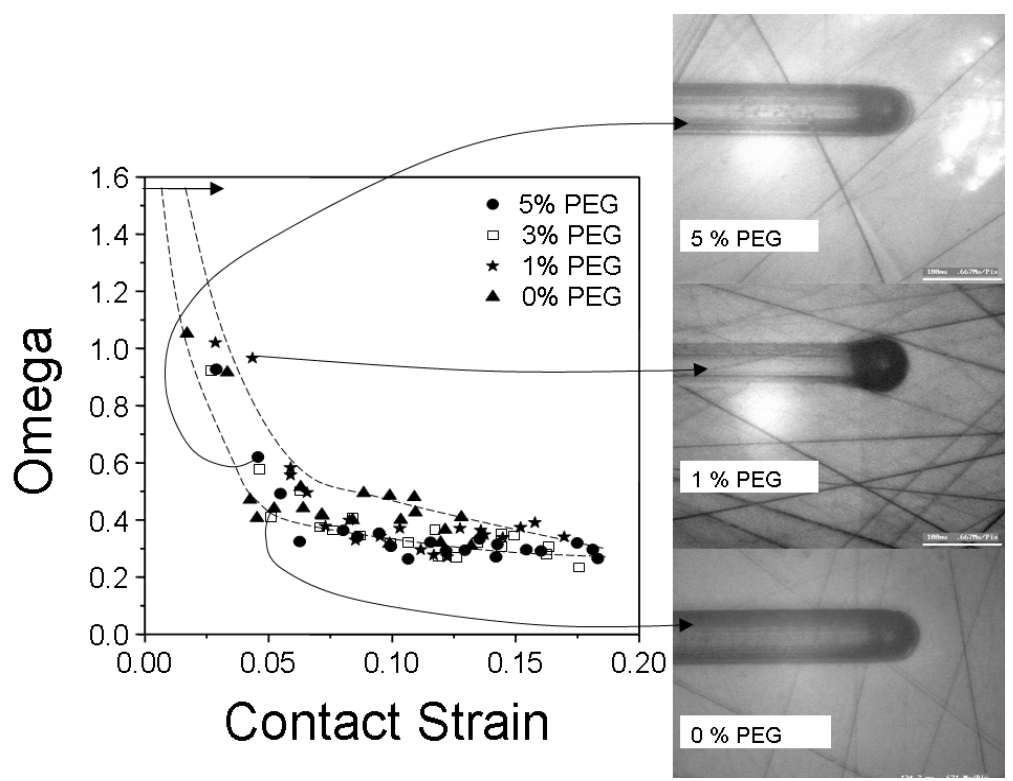

Figure 7: Variation of omega with contact strain and the percentage of PEG for PMMA at room temperature (sliding/scratching speed $=0.03 \mathrm{~mm} / \mathrm{sec}$, tip radius $=116 \mu \mathrm{m})$.

PMMA at contact strain about 0.05 . On the other hand, it is viscoelastic for crodamide-plasticized samples at the same value of contact strain (Figure 6).

It is interesting to note that at the same conditions of temperature and contact strain, the nature of contact for PEG plasticized samples of PMMA is similar to that of pure PMMA, i.e. almost plastic (Figure 7). The values of the contact angle for PEG plasticized samples also remain almost same as that of pure PMMA for a given value of contact strain. The little decrease in friction for the samples containing $1 \%$ PEG is also reflected in the nature of contact as is clear from the in-situ photograph and the value of contact angle is also increased a little bit correspondingly. However, at a given condition of temperature and normal load, the contact is purely elastic for all samples at very low contact strains and is purely plastic at very high values of contact strain (Figures 6 and 7).

\subsection{Bulk properties}

Elastic Modulus and Yields Stress are the bulk material properties affecting the scratch process.

\subsubsection{Elastic modulus}

Elastic modulus displays a principal part in the scratch process, particularly for the duration of the indentation stage. According to the scratch model, the Young's modulus, scratching tip geometry, and Poisson's ratio directly decide the range of the contact zone during scratch. For polymers with lower modulus, the 
contact zone dimension increases a lot with decreasing modulus. It is fascinating to note that for polymers with high Young's modulus, the consequence of the change in the Young's modulus on the contact zone size becomes immaterial.

The penetration depth is more responsive to the modulus when it is inferior to $1 \mathrm{GPa}$. The maximum tensile stress is on the surface immediately at the rear of the scratcher during scratch. It is clear that polymers with higher moduli demonstrate a higher maximum tensile stress on the surface. This may result in the development of surface cracks, crazes, cavitations, and debonding between phases of multiphase polymers. As mentioned above, the Young's modulus plays an important role in generating scratch damage. A certain level of stiffness in polymers can assist to maintain a low scratch depth if the polymer is inclined to plastic flow scratch damage. In contrast, polymers with elevated rigidity induce high stress concentration during scratch, which could prop up the creation of surface cracks, crazes, cavitations, and debonding. In this case, it is preferable to lower the modulus or enhance the ductility of the polymer [3].

As the percentage content of plasticizer in all cases was not so high, their consequence on the mechanical properties is not up to significant value (Figure 8). Both crodamide and PEG have no remarkable effect on major bulk properties like Young's modulus. However, PEG disturbs these properties comparatively more than does crodamide. This difference of bulk behaviour can be attributed to the enhanced tendency of migration of crodamide from bulk to the surface of the material as is discussed in previous section. Due to higher molecular weight of PEG, it migrates to the surface a little and mainly remains inside the bulk of material and hence disturbs the bulk behaviour more pronouncedly as compared to the crodamide.
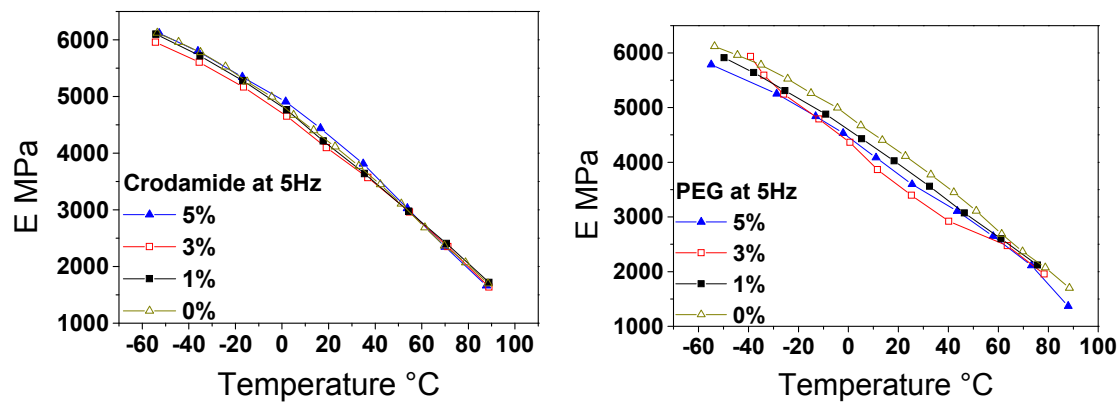

Figure 8: $\quad$ Effect of crodamide and PEG on young's modulus of PMMA.

\subsubsection{Yield stress}

The yield stress plays a prime role to determine the upper boundary of the mean contact pressure and the size of the plastic zone. Polymers having an elevated yield stress are subjected to smaller plastic flow scratch damage. On the other hand, cracks are generally generated on the scratch surface if the utmost tensile stress on the surface exceeds the tensile strength and if the tensile strength is lesser than the yield stress of the polymer. Therefore, the tensile strength of the 
polymer plays a key role in determining the growth of either the plastic flow scratch pattern or the brittle fracture scratch pattern on the scratch surface [3].

The effect of both crodamide and PEG on nominal stress and nominal strain at break is not very clear. Generally speaking, both increase these values a little bit for PMMA and this is more pronounced in case of PEG (Figure 9). However, at low temperature, crodamide first decreases the nominal stress and strain at break of PMMA but further increase in its content again increase the nominal stress and strain values of PMMA. Similarly the effect of both Crodamide and PEG on shear stress and shear strain at breakage is not very clear (Figure 10). Shear stress and strain at breakage is affected a little by both the crodamide and PEG and this effect is more significant in case of crodamide. However, at higher temperature, this effect is more pronounced for PEG.
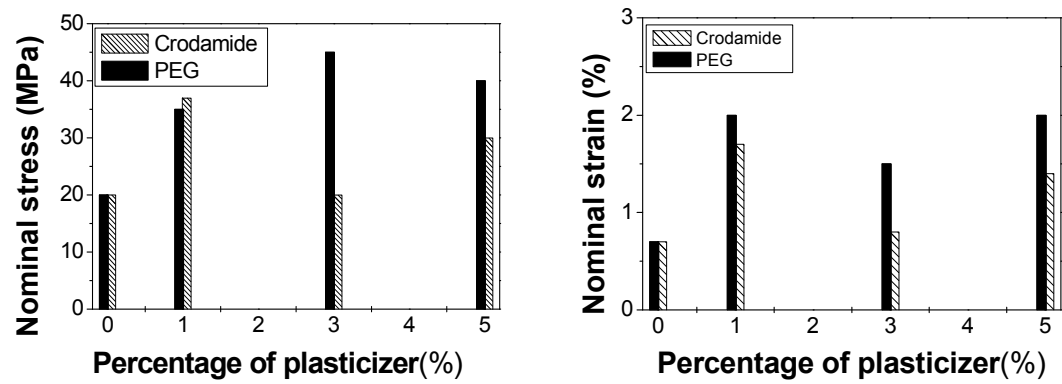

Figure 9: Effect of crodamide and PEG on nominal stress and strain at break of PMMA at $40^{\circ} \mathrm{C}$ (traction speed $=10^{-4} / \mathrm{sec}$ ).
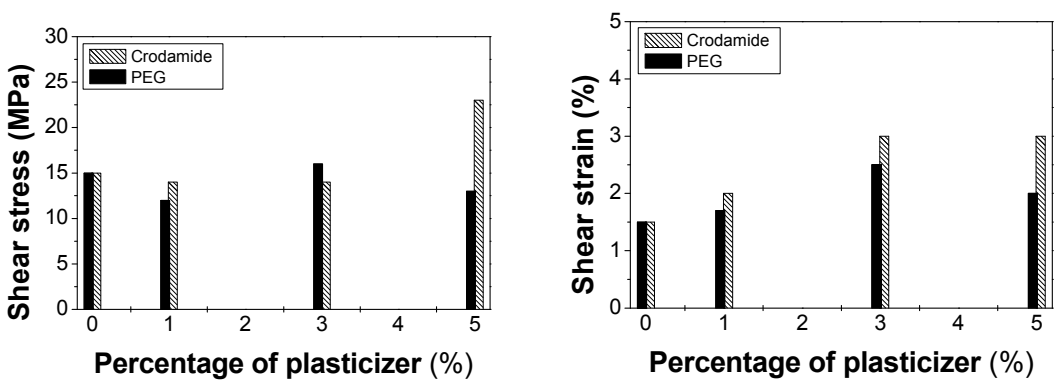

Figure 10: Effect of crodamide and PEG on shear stress and strain at break of. PMMA at $40^{\circ} \mathrm{C}$ (traction speed $=10^{-4} / \mathrm{sec}$ ).

\section{Conclusions and prospective}

The following conclusions can be drawn:

1. The coefficient of friction of PMMA can be decreased by the use of appropriate plasticizer. 
2. The crodamide is more effective than PEG for reducing the friction coefficient of PMMA and the decrease in friction coefficient is proportional to the crodamide content.

3. The decrease in friction coefficient of PMMA by crodamide is not at the cost of bulk properties. They remain almost unaffected.

4. The decrease in friction is associated with the nature of the contact between the tip and the polymer surface.

5. The use of crodamide is also favourable as it improves the bulk properties a little bit along with improving the surface properties in contrast to PEG, which reduces the bulk properties a little bit.

6. Generally speaking, nominal stress and nominal strain at break are increased by both crodamide and PEG but at low temperatures it is decreased a little bit by crodamide.

7. Similarly, shear stress and shear strain at break are increased by both crodamide and PEG.

However, further work is indispensable to enumerate the well balanced and optimised study surrounded by the upgrading of the surface behaviour (like reduction in friction coefficient) and the subsequent change in bulk properties.

\section{Acknowledgements}

The financial support provided by the Higher Education Commission, Pakistan is gratefully acknowledged. The authors would thank and express their sincere appreciation to Mr. Favier Damien for his help during the experimental work.

\section{References}

[1] Hamouda, A.M.S., The influence of humidity on the deformation and fracture behaviour of PMMA. Journal of Materials Processing Technology, 124(1-2): p. 238-243. 2002.

[2] Wong, M., et al., A new test methodology for evaluating scratch resistance of polymers. Wear, 256(11-12): p. 1214-1227. 2004.

[3] Xiang, C., et al., Scratch behavior and material property relationship in polymers. Journal of Polymer Science Part B: Polymer Physics, 39(1): p. 47-59. 2001

[4] Gauthier, C. and R. Schirrer, Time and temperature dependence of the scratch properties of poly(methylmethacrylate) surfaces. Journal of Materials Science, 35(9): p. 2121-2130. 2000.

[5] Pelletier, H., et al., Viscoelastic and elastic-plastic behaviors of amorphous polymeric surfaces during scratch. Tribology International, 41(11): p. 975984. 2008.

[6] Jardret, V. and P. Morel, Viscoelastic effects on the scratch resistance of polymers: relationship between mechanical properties and scratch properties at various temperatures. Progress in Organic Coatings, 48(2-4): p. 322-331. 2003.

[7] Adams, M.J., et al., An experimental study of the nano-scratch behaviour of poly(methyl methacrylate). Wear, 251(1-12): p. 1579-1583. 2001. 
[8] Tanglumlert, W., et al., Hard-coating materials for poly(methyl methacrylate) from glycidoxypropyltrimethoxysilane-modified silatrane via a sol-gel process. Surface and Coatings Technology, 200(8): p. 2784-2790. 2006.

[9] Kopesky, E.T., G.H. McKinley, and R.E. Cohen, Toughened poly(methyl methacrylate) nanocomposites by incorporating polyhedral oligomeric silsesquioxanes. Polymer, 47(1): p. 299-309. 2006.

[10] Gauthier, C., S. Lafaye, and R. Schirrer, Elastic recovery of a scratch in a polymeric surface: experiments and analysis. Tribology International, 34(7): p. 469-479. 2001.

[11] Briscoe, B.J., et al., Scratching maps for polymers. Wear, 200(1-2): p. 137147. 1996.

[12] Sujeet K Sinha, B.J.B. Polymer Tribology. 2009.

[13] Sinha, S.K. and D.B.J. Lim, Effects of normal load on single-pass scratching of polymer surfaces. Wear, 260(7-8): p. 751-765. 2006.

[14] Felder, E. and J.-L. Bucaille, Mechanical analysis of the scratching of metals and polymers with conical indenters at moderate and large strains. Tribology International, 39(2): p. 70-87. 2006.

[15] Briscoe, B.J. and S.K. Sinha, Scratch Resistance and Localised Damage Characteristics of Polymer Surfaces - a Review. Materialwissenschaft und Werkstofftechnik, 34(10-11): p. 989-1002. 2003.

[16] Jardret, V., et al., Understanding and quantification of elastic and plastic deformation during a scratch test. Wear, 218(1): p. 8-14. 1998.

[17] Gauthier, C., et al., Scratching of a coated polymer and mechanical analysis of a scratch resistance solution. Tribology International, 39(2): p. 88-98. 2006.

[18] Lafaye, S., C. Gauthier, and R. Schirrer, A surface flow line model of a scratching tip: apparent and true local friction coefficients. Tribology International, 38(2): p. 113-127. 2005.

[19] Lafaye, S., C. Gauthier, and R. Schirrer, Analysis of the apparent friction of polymeric surfaces. Journal of Materials Science, 41(19): p. 6441-6452. 2006.

[20] Subhash, G. and W. Zhang, Investigation of the overall friction coefficient in single-pass scratch test. Wear, 252(1-2): p. 123-134. 2002.

[21] Scott, M.P., M. Rahman, and C.S. Brazel, Application of ionic liquids as low-volatility plasticizers for PMMA. European Polymer Journal, 39(10): p. 1947-1953. 2003.

[22] Wypych, G., ed. Handbook of Antiblocking, Release and Slip Additives; 2005.

[23] http://www.atskimya.com/msds/spect59.pdf.

[24] http://www.thefreelibrary.com/Lubricants+and+processing+aids.a0189051 619.

[25] Markarian, J., Slip and antiblock additives: surface medication for film and sheet. Plastics, Additives and Compounding, 9(6): p. 32-35.2007

[26] Lafaye, S., C. Gauthier, and R. Schirrer, Analyzing friction and scratch tests without in situ observation. Wear, 265(5-6): p. 664-673. 2008. 